\title{
Electron/Photon Reconstruction Performance with the ATLAS Detector
}

\author{
K. Benslama, on behalf of the ATLAS Collaboration \\ Physics Department, University of Regina, Saskatchewan, Canada
}

\begin{abstract}
The calibration of electromagnetic clusters is one of the key issues of ATLAS (and the LHC) in 2007. The cluster algorithm starting from electronically calibrated calorimeter cells will be described. Local position and local energy variations are corrected for. As a last step, longitudinal weights are applied to correct for energy loss upstream of the calorimeter. Strongly influenced by testbeam studies, the new longitudinal weighting optimizes simultaneously energy resolution and linearity. Methods to derive the final calibration parameters from the physics events have been developed. The electron and photon identification methods and their performance will then be discussed.
\end{abstract}

Keywords: Identification of electron and photon

PACS: $29.40 . \mathrm{Vj}$

\section{INTRODUCTION}

The Large Hadron Collider (LHC) at CERN is a proton-proton collider with $14 \mathrm{TeV}$ energy in the center of mass and a design luminosity of $10^{34} \mathrm{~cm}^{-2} \mathrm{~s}^{-1}$. The ATLAS experiment is one of the two major multi-purpose detectors currently under construction at the LHC. Its inner detector consists of tracking detectors enclosed in a solenoidal magnet with $2 \mathrm{~T}$ field. From the inner radius to the outside radius, it consists of pixel detectors, silicon strip detectors (SCT) and transition radiation drift tubes (TRT), covering the pseudo-rapidity region $|\eta|<2.5$.

The inner detector is surrounded by a sampling electromagnetic calorimeter based on lead and liquid argon (LAr) technology and a hadronic calorimeter based on LAr in the end-caps and iron/scintillator tiles in the barrel. The global detector dimensions are defined by a large air-core muon spectrometer, providing precision measurements of high- $p_{T}$ muons over $|\eta|<2.5$.

The LHC physics program [1] ranges from the search for the Higgs boson, searches for physics beyond the standard model, such as supersymmetric particles, new additional $\mathrm{W}$ and $\mathrm{Z}$ bosons, and also precision studies, such as measurements of the t quark and $\mathrm{W}$ boson masses and unexpected signals from unpredicted physics scenarios.

\section{ELECTRONS AND PHOTONS RECONSTRUCTION AND IDENTIFICATION}

Events with electrons and photons in the final state are important signatures for many physics analyses at the LHC, since electrons and photons are relatively easy to measure 
precisely and to trigger on. Isolated high $p_{T}$ electrons and photons are not easy to identify at the LHC because of the large QCD background from high- $p_{T}$ jets, which results in an electron/jet ratio of about $10^{-5}$ at the LHC (to be compared to about $10^{-3}$ at the Tevatron) for isolated electrons from $\mathrm{W} / \mathrm{Z}$ decays, and to a photon/jet ratio of $10^{-4}$ (to be compared to about $10^{-3}$ at the Tevatron). Final states containing electrons or photons such as $H \rightarrow 4 e$ or $H \rightarrow \gamma \gamma$ decays provide convincing discovery channels [1].

Electrons and photon reconstruction mainly uses data coming from the electromagnetic calorimeter (EM) and the inner detector (ID) systems. Electromagnetic objects can be identified by looking at the transverse and longitudinal shower shapes and at isolation variables. For electrons, a track is then required to match in position and energy that measured in the electromagnetic calorimeter. For photons, no track is required (except in the frequent case of converted photons) but $\gamma / \pi^{0}$ separation criteria is required using some of the unique features of the electromagnetic calorimeter.

\section{Energy Measurement}

Four longitudinal samplings are available over most of the EM calorimeter coverage for the energy measurement. Where available, the presampler is used to correct for the energy lost in the upstream material. The strip section provides information on the early part of the shower, besides contributing to the energy measurement. Most of the shower energy, however, is deposited in the middle section sampling, while the back sampling is used for high energies.

The energy response of the calorimeter is potentially affected by the following effects:

- upstream energy losses;

- $\phi$-modulations and azimutal gaps between presampler sectors;

- lateral leakage outside the cluster;

- longitudinal leakage behind the EM calorimeter.

Efficient corrections are applied for most of these effects, which allows to preserve a good energy resolution.

In general, the energy reconstructed in the EM calorimeter can be written as:

$$
E_{\text {tot }}=W_{\text {glob }}\left(b+w_{p s} E_{p s}+E_{s t r}+E_{\text {mid }}+E_{\text {back }}\right)
$$

Where $w_{g l o b}$ is a global calibration factor, $\mathrm{b}$ is an offset with units of energy, motivated by recent test beam analyses. This offset was found to optimize simultaneousely electron energy linearity and resolution. $E_{p s}, E_{s t r}$, and $E_{\text {back }}$ are the energies measured in a given cluster of cells in the presampler, strip section, middle and back samplings of the calorimeter respectively. The mean fractional energy deviation from truth (data points) and the energy resolution (error-bars) for $100 \mathrm{GeV}$ electrons after application of longitudinal weights is shown in Fig.1. 

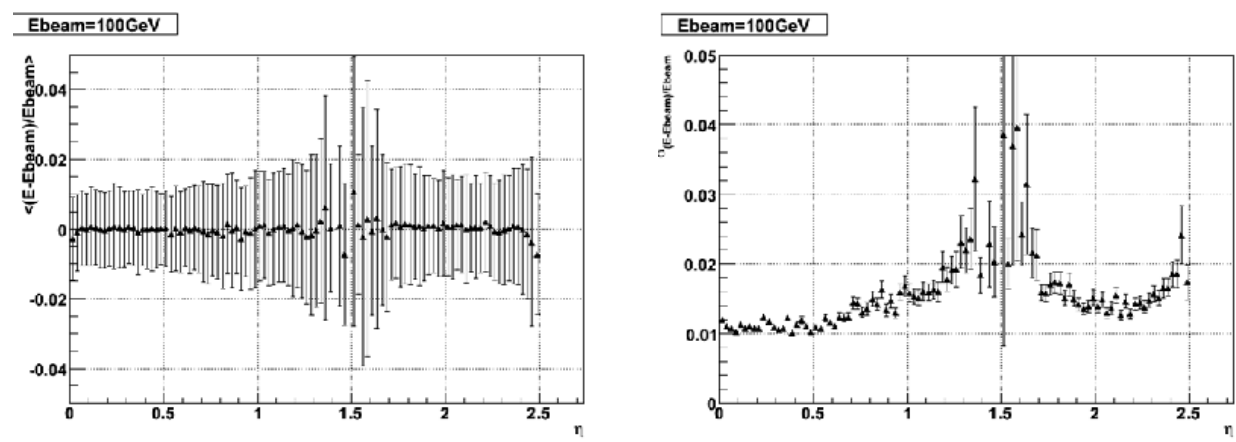

FIGURE 1. The mean fractional energy deviation from truth (data points) and the energy resolution (error-bars) for $100 \mathrm{GeV}$ electrons after application of longitudinal weights.

TABLE 1. Electron identification efficiency $\varepsilon_{e}$ for single electrons with $p_{T}>25$ $\mathrm{GeV}$ and jet rejection of the offline analysis at low luminosity [2].

\begin{tabular}{lrr}
\hline & $\varepsilon_{e}(\%)$ & rejection $\left(\times 10^{3}\right)$ \\
\hline Calo & $91.2 \pm 0.4$ & $1.3 \pm 0.1$ \\
ID & $81.3 \pm 0.5$ & $19.5 \pm 2.5$ \\
ID-Calo & $76.4 \pm 0.6$ & $57.0 \pm 7$ \\
\hline
\end{tabular}

\section{Electron/jet Separation in ATLAS}

The identification of isolated electrons with $p_{T}>20 \mathrm{GeV} / \mathrm{c}$ will be essential for many physics searches at the LHC. A challenging task will be to identify electrons in the presence of high QCD backgrounds, which is $\sim 10^{5}$ times higher, as in the case of $\mathrm{W}$ and top decays.

To separate electrons from jets, cuts were developed to maintain a reasonable electron identification efficiency even with pile-up at high luminosity, while removing a high fraction of jets events. The cuts include Level-1 and High Level Trigger cuts, shower shape and isolations cuts in the calorimeter, cuts on track in ID, cuts on ID-Calo matching in position and energy and TRT cuts. The effect of applying all these cuts one after the other to both single electrons and jets samples is shown in Tab. 1. After calorimeter selection, the dominant background consists of photons from $\pi^{0}$ and $\eta$ decays. This is significantly reduced by requiring the presence of a high $p_{T}$ track. After the ID-Calo match, charged hadrons remain the main background. The signalto-background ratio is 2:1 for a QCD-jet rejection of $0.6 \times 10^{5}$. The signal is equally from semileptonic decays of heavy quarks and isolated electrons from $\mathrm{W}$ and $\mathrm{Z}$ decays. The QCD jet rejection can be improved by using the transition radiation rejection of the TRT. An electron efficiency of about $70 \%$ is obtained while a QCD jet rejection above $10^{5}$ can be achieved. Finally, removal of photon conversions by direct reconstruction, would allow the identification of a pure electron inclusive sample with a jet rejection of around $10^{6}$. Further improvements can be achieved by using multivariate techniques. 


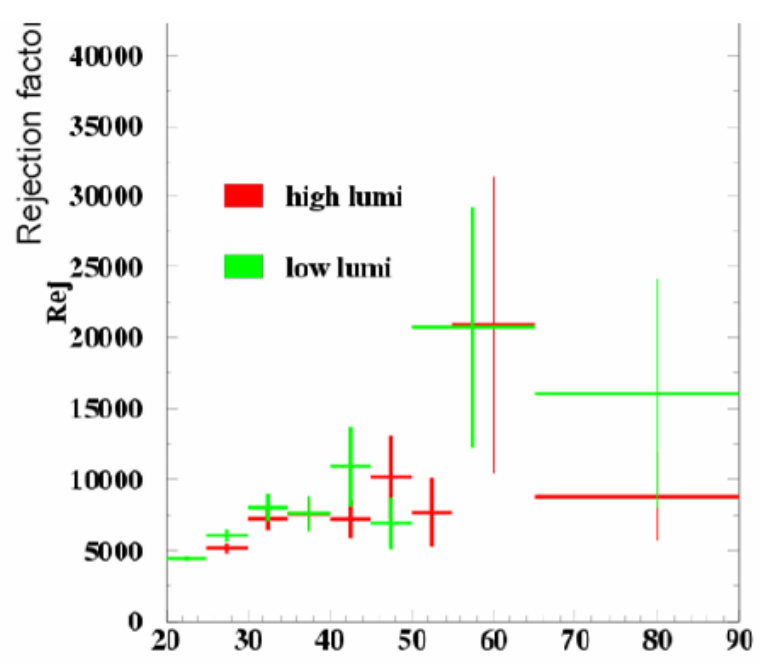

FIGURE 2. Jet rejection as a function of the transverse energy of the jet for a photon identification efficiency of $80 \%$ at low and high luminosity [3].

\section{Photon/jet Separation in ATLAS}

Given the amount of material in front of the calorimeter, about $40 \%$ of the photons from e.g $H \rightarrow \gamma \gamma$ decays convert into $e^{+} e^{-}$pairs before depositing their energy in the calorimeter. Since the $H \rightarrow \gamma \gamma$ is small, it is important to recover these conversions to maintain its efficiency as high as possible. Conversions are first searched for in the Inner Detector, and the energy deposited in the calorimeter in a $3 \times 7 \eta \times \phi$ window is computed, since this window size allows to recover a significant fraction of the initial photon energy. Using an estimated dependence between the conversion radius, $E_{T}$ and the photon energy, it is possible to reconstruct the initial photon energy.

If the photon has not been reconstructed as a conversion, the photon/jet separation relies on the search for electromagnetic objects, with cuts including the Level-1, High Level Trigger cuts, shower shapes and isolation cuts in the calorimeter, with the requirement that no track is found in the ID within a $\Delta \eta \times \Delta \phi$ region of size $\pm 0.1 \times \pm 0.1$ around the calorimeter cluster. Fig. 2 shows the jet rejection after photon selection cuts as a function of the jet transverse energy $E_{T}$. A rejection factor better than 7000 can be obtained for $E_{T}>40 \mathrm{GeV}$, both for low and high luminosity.

\section{Electron/pion Separation in ATLAS}

The efficient tagging of low energy electrons is an important tool for B-physics, as well as a complementary method to b-tagging. Separating low energy electrons from pions by analysing the energy deposits in the calorimeter alone is not an easy task because these electrons are within or near to jets. A better option is to use the ID to seed the calorimeter clustering. This strategy consists of several steps. First, tracks with $p_{T}>2 \mathrm{GeV}$ are found in the ID and then one looks for the EM calorimeter regions hit 


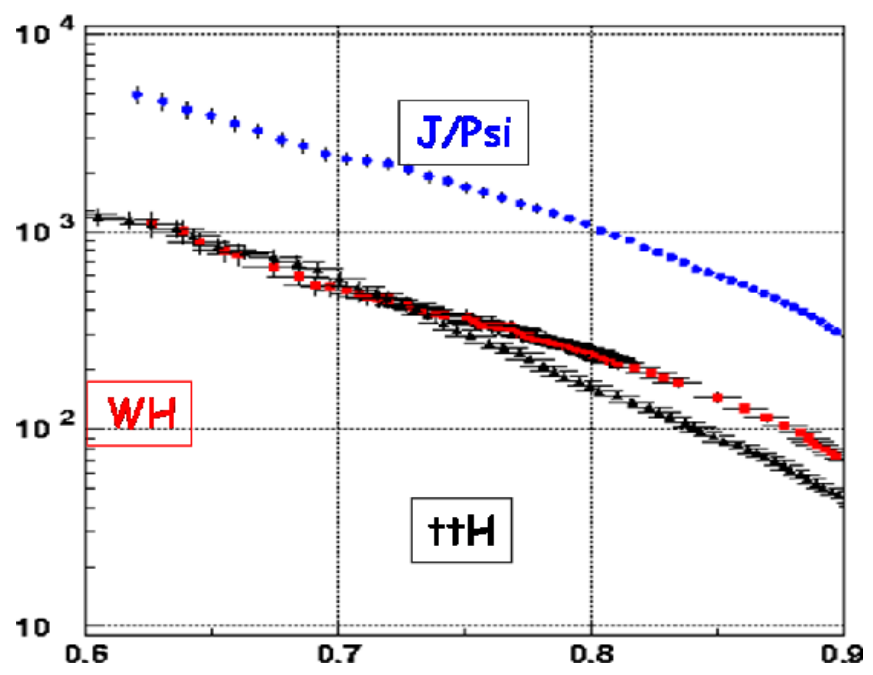

FIGURE 3. Pion rejection as a function of electron identification efficiency for low energy electron [4] [5].

by the tracks. By combining various shower shapes estimators and the E/P value and the information from the TRT, it is possible to get the pion rejection versus the electron identification efficiency curves of Fig. 3.

For the $\mathrm{J} / \psi$ sample, a pion rejection factor of 1000 is achieved for an electron identification efficiency of $80 \%$. Electrons from $W H \rightarrow b \bar{b}$ events are located inside jets. Thus, their identification is more difficult. For $80 \%$ electron identification efficiency, the pion rejection factor is $\sim 250$. This soft electron identification could then be used for b-tagging as a complementary method to the standard vertex-based tagging.

\section{CONCLUSION}

The ATLAS collaboration has developed powerful electron and photon identification algorithms and tuned them over the past years on detailed Monte Carlo simulation. While maintaining high electron and photon identification efficiency, these algorithms achieve a very high QCD jet rejection. This strong identification ability coupled with a good performance of the ATLAS detector are crucial for many discovery channels.

\section{ACKNOWLEDGMENTS}

The author is greatful to the ATLAS Collaboration.

\section{REFERENCES}

1. ATLAS detector and physics performance technical design report, ATLAS TDR $14 \& 15$, CERN/LHCC/99-14 \& 15. 
2. F. Derue, C. Serfon, "Electron/jet separation with DC1 data", ATLAS Note ATL-COM-PHYS-2005016.

3. M. Escalier et al., "Photon/jet separation with DC1 data", ATLAS Note ATL-COM-PHYS-2005-048.

4. T. Bold et al., "Pile-up studies for soft electron identification and b-tagging with DC1 data", ATLAS Note ATL-COM-PHYS-2005-027.

5. F. Derue et al., "Reconstruction of DC1 $J / \psi \rightarrow e^{+} e^{-}$decays and use for low energy calibration of the ATLAS electromagnetic calorimeter", ATLAS Note ATL-COM-PHYS-2005-022. 